\title{
STUDY OF RADOME OBSTRUCTIONS ON ANTENNA PERFORMANCE: LIGHTNING STRIPS AND NOSE RADOME EFFECTS
}

\author{
Anas Khan \\ B.E. Electronics \& Communication \\ MSc. Physics \\ Birla Institute of Technology \& Science, Pilani
}

\begin{abstract}
This review paper investigates the impact of lightning segmented diverter strips on radome-antenna performance. A brief overview is given and the admissible length of strips is calculated.
\end{abstract}

The effects of using a nose radome on the antenna performance is also calculated. Three models, namely Physical Optics (PO) model and two computational models, FEKO and Shako, that work using Method of Moments are used and Radiation pattern results are compared

\section{INTRODUCTION}

In the first section, we look at the lightning strike protection of radomes using lightning diverter strips and decide the necessary characteristics of the strip for optimum performance.

According to the statistics, every year around one commercial aircraft is struck by lightning [1]. Commercial aircrafts carry a lot of people. Research shows that around 575 people are present on average in a passenger aircraft and planes like the airbus A380 can carry as many as 900 people at once.

Communication/antenna problems may result in accidents that may risk the life of these many people. Thus, it is really necessary to ensure that the aircraft equipment does not fail under lightning strikes or other weather circumstances.

There are two types of lightning strikes to the aircrafts. First is when the aircraft itself causes the discharge of electricity being inside the intense electrostatic field of the thundercloud. Second, when the aircraft intercepts a leader that approaches it. The mechanism for lightning discharge can be explained using the "bidirectional leader theory" [3].
In order to function in an appropriate manner, all the aerial devices, from satellite antenna to radar systems need to be located external to the aircraft metallic body. However, antennas have an aerodynamic structure that provides hindrance to the smooth flight of the aircraft and as a result need a transparent EM structure to protect them from airflow forces [1]. These Electromagnetic transparent structures are referred to as Radomes and are made of dielectric material. These radomes provide EM screening effect to the electrical lightning discharges which may occur from the thunderclouds. Thus, the lightning protection system of the radomes should carry out two purposes: Firstly, they need to protect the radar antenna system from puncturing by lightning strikes. Secondly, they need to be designed in such a manner that they do not propose significant attenuation to the transmission or receiving signal of the antenna.

For this purpose, lightning diverter strips are designed and placed on the outer surface of the radome either as a solid metal strip or as conductive buttons placed closely in a series.

In the Second section, we look at the modelling effects of nose radome on the antenna performance. We look at two methods for assessing the effects of nose radome on the radar performance.

First, the improved Physical Optics method that takes into account the radome thickness and multiple layers of the radome. Second, the fullwave methods known as the FEKO and SHAKA the take into account the attenuation due to the multiple reflections and thus produce really accurate results. The models are applied to predict the radiation pattern of a radar antenna behind a generic nose radome and behind the radome of a fighter aircraft [2]. Later we also discuss the applicability of the two models. 


\section{LIGHTNING ZONES \& PROTECTION DESIGN}

For the purpose of protection design, there are certain lightning zones that have been defined. These zones identify where the lightning is likely or unlikely to attach and also other regions of the aircraft, where the attachment would be for a very short duration of time. These zones can vary in aircrafts because they depend on the geometry, design, aerodynamics, materials etc. of the aircraft. A recent study showed that majority of the lightning discharges are caused by the aircraft itself. This shows that the extremities of the aircraft with high curvatures serve as regions of high electric field that initiate the discharge.

It also has been shown (Petrov \& Waters, 1994,1995) that the electric field needed to initiate lightning discharge at $4 \mathrm{Km}$ altitude is half of the value at sea level. Therefore, the risk of striking increases with higher altitudes.

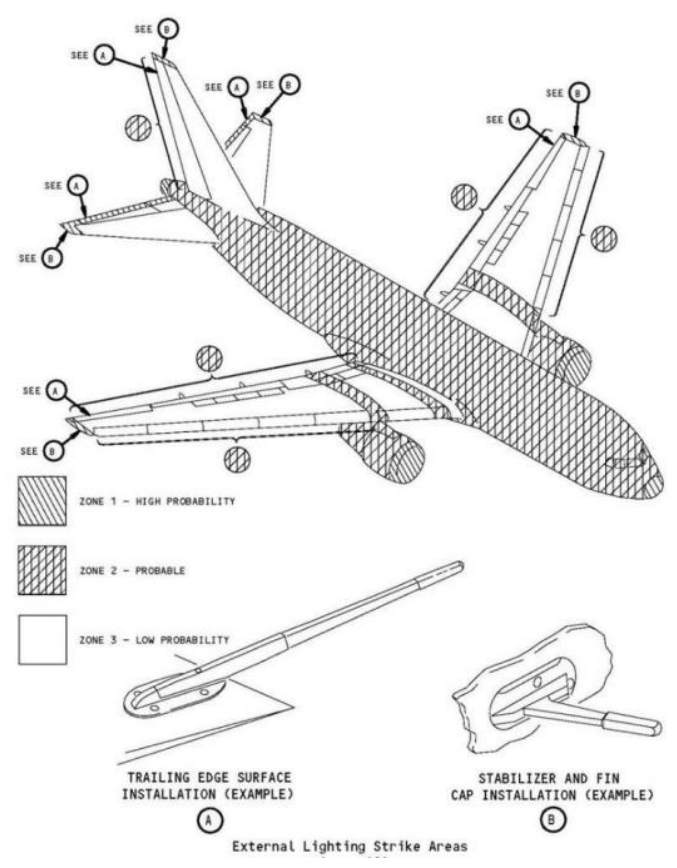

The mechanical design of the radome wall that is the front part of the aircraft is generally of two types: solid(monolithic) or sandwich type. The solid radome usually is made up of resins with reinforcements that minimize the loss whereas the sandwich type radome consists of alternating layers of high-density and low-density materials sandwiched together.

\section{A. Diverter Strips}

The radar and telecom antenna in aircrafts are usually located at the nose of the radomes and made up of non-metallic materials, making it a high probability region of lightning strike. To counter this problem, diverter strips were made. These strips screen the electric field on the surface of the antenna that have been induced by lightning. These strips are of two types:

- Solid

- Segmented

\section{B. Solid Strips}

The solid strips are basically made of permanent conductor that extend beyond the antenna. They shield the antenna from the induced electric field by collapsing the field inside the radome. These types of strips can withstand multiple lightning strikes.

They are usually used for commercial aircraft applications. They are usually installed in zone $1 \mathrm{~A}$ of the aircraft but depends on the lightning current load. The area of the strips depends on the type of material of the strip. These are attached to the aircraft with the help of bolts.

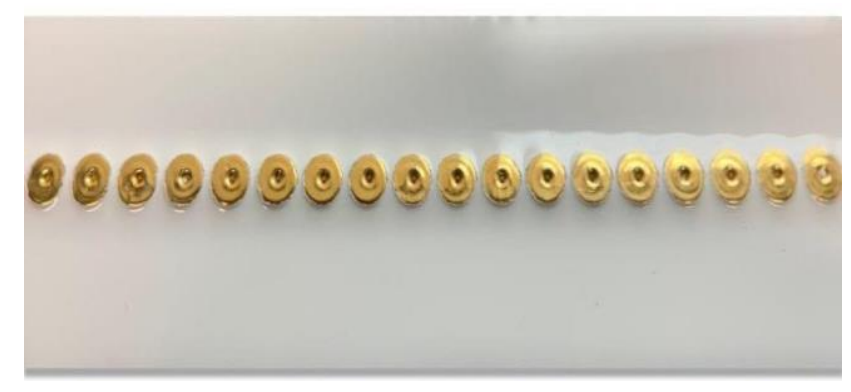

\section{Segmented Strips}

A segmented strip is a thin strip of closely spaced metal disc with gaps in between them. These gaps attract the lightning away from sensitive components such as the radome.

These strips are used where effect on the radiation pattern of the antenna by the lightning discharge is more significant to consider. These strips have limited multistrike capability [1].

In [1], two different types of segmented diverter strips have been considered. The diameter of the first strip button is $0.76 \mathrm{~mm}$ and of that of the second one is $1.52 \mathrm{~mm}$ [1].

The primary effectiveness of these diverter strips can be obtained if we observe their ignition voltage as a function of the strip length. The 


\section{International Journal of Engineering Applied Sciences and Technology, 2021 \\ Vol. 6, Issue 2, ISSN No. 2455-2143, Pages 209-212 \\ Published Online June 2021 in IJEAST (http://www.ijeast.com)}

measurements were performed with two high voltage waveforms A and D[4]. The mean ignition voltage has been plotted in [1].

The mean ignition voltage was observed to increase roughly with increasing diverter strip length. On estimating the admissible length of the diverter strip based on this relation, $85 \mathrm{~cm}$ was considered to be a good approximation for the lower limit of the diverter strip of $1.52 \mathrm{~mm}$ sized buttons.

\section{H.V. TESTS OF RADOME-ANTENNA SYSTEM}

In order to predict the optimum length of the diverter strips, an experimental test set-up was made which took into account the metallic antenna platform [1]. The test set-up is shown in [1].

Result: None of the tests with the high-voltage waveforms $\mathrm{A}$ and $\mathrm{D}$ with both polarities resulted in puncture of the sandwich radome up to a length of about $100 \mathrm{~cm}$. Thus for $1.52 \mathrm{~mm}$ button sized segmented diverter strips, $100 \mathrm{~cm}$ could be used as an acceptable length of strip

\section{SECTION II}

\section{MODELLING EFFECTS OF NOSE RADOME ON ANTENNA PERFORMANCE}

Fixed- wing aircrafts which are typically contain radars for weather detection use the nose type radomes. A radome is often used to prevent the accumulation of ice and rain on the surface of the radome directly, thus serving as a shield for the antenna surface. Several military grade aircrafts use the nose shaped radomes as well.

In the paper [2], two computational models have been used. First is the improved Physical Optics model that takes into consideration multiple radome layers and radome wall thickness. Second is the Full Wave

Solver which solve the Maxwell and Stratton-Chu equations using Method of Moments or Finite Element Method (FEM).

For the application of these above methods, the antenna should be subdivided into triangular or quadrilateral elements. The computational resources required for the High Frequent processes require a time complexity of $O(N)$. That is why the Physical Optics method is really convenient for tasks where successive simulations are required. However, this method has certain disadvantages. It does not take into account the attenuation of the reflected signals.
The other methods that use the Method of Moments require a time complexity of $O\left(N^{\wedge} 3\right)$ which would mean exhaustive computations. In order to reduce this complexity problem, methods like the Fast Multipole expansions and Multilevel schemes were introduced which reduced the time complexity to $O(N \log N)$ [2]. The two models we use are the FEKO software package of Altair [4]. The other is the developed code for Shako.

We discuss the differences in the radiation pattern of all three methods of computation.

\section{RESULTS OF SIMULATION}

It is observed that after the application of the farfield equations to the corresponding three models we obtain solution of radiation patterns mentioned in [2]. There is a very good comparison between the FEKO and Shako methods and the Physical Optics (PO) method also occurs in good agreement. There are some differences in the PO method.

Due to neglecting the reflection attenuation, the image lobe was found to be $1-2 \mathrm{~dB}$ higher than those computed by the other two models. This is because it takes into account only one reflection. Apart from the image lobe, the main lobe values only differed by a small amount. Hence, there is a valid comparison between the radiation model computed by the three models.

\section{CONCLUSIONS}

In Section I, the electric field strength inside a dielectric radome is not reduced significantly even if the radome is painted with anti-static paint [1]. Thus, to avoid the discharge from the radar inside the radome, dielectric strips are used to produce the necessary electromagnetic screening and shielding effect. A hybrid lightning protection system consisting of the solid and segmented strips is being devised by Airbus Défense [1].

In Section II, the three computational methods were applied to the generic radome with slotted antennas. Results of the two Method of Moments methods, FEKO and Shaka are applied and compared to the Physical Optics method. Comparison between the radiation pattern of the three methods show good agreement. Apart from the image lobe, the main beam attenuation and deflection are estimated quite accurately [2].

\section{REFERENCES}

[1] Karch, Christian \& Calomfirescu, M \& Rothenhaeusler, Markus \& Brand, C \& Meister, 
Heinz. (2017). FFS: LIGHTNING STRIKE PROTECTION OF RADOMES - AN OVERVIEW.

[2] J. L. Rotgerink, H. van der Ven, T. Voigt, E. Jehamy, M. Schick and H. Schippers, "Modelling of effects of nose radomes on radar antenna performance," 2016 10th European Conference on Antennas and Propagation (EuCAP), 2016, pp. 1-5, doi: 10.1109/EuCAP.2016.7481773.

[3] N.I. Petrov ${ }^{1}$, A. Haddad ${ }^{2}$, G.N. Petrova ${ }^{1}$, H. Griffiths ${ }^{2}$ and R.T. Waters ${ }^{21}$ Istra, Moscow region,

${ }^{2}$ Cardiff University, ${ }^{1}$ Russia ${ }^{2}$ United Kingdom

[4] EUROCAE ED-84, , European Organisation for Civil Aviation Equipment, Paris 1997, SAE ARP5412-Revision, "Aircraft

Lightning Environment and Related

Test Waveforms," SAE Aerospace, 2005 